\title{
Analysis and variability of TGF $\beta$ measurements in cancer patients with skeletal metastases
}

\author{
Peter J O’Brien' \\ Rajeev Ramanathan ${ }^{2}$ \\ Jonathan M Yingling' \\ Jose Baselga ${ }^{3}$ \\ Mace L Rothenberg ${ }^{4}$ \\ Michael Carducci ${ }^{5}$ \\ Thomas Daly' \\ Dorothy Adcock ${ }^{2}$ \\ Michael Lahn' \\ 'Eli Lilly and Company Research \\ Laboratories, Lilly Corporate Center, \\ Indianapolis, IN, USA; ${ }^{2}$ Esoterix \\ Coagulation, Aurora, CO, USA; \\ ${ }^{3}$ Vall d'Hebron University Hospital, \\ Barcelona, Spain; ${ }^{4}$ Vanderbilt-Ingram \\ Cancer Center, Vanderbilt University, \\ Nashville, TN, USA; ${ }^{5}$ The Sidney \\ Kimmel Comprehensive Cancer \\ Center, Johns Hopkins University, \\ Baltimore, MD, USA
}

Correspondence: Michael Lahn Lilly Deutschland GmbH,Werner-Reimers Strasse 2-4, 61352 Bad Homburg, Federal Republic of Germany

Tel +496172 2732052

Fax +4961722732190

Emailmlahn@lilly.com

\begin{abstract}
Transforming growth factor beta (TGF $\beta$ ) plays an important role in cancer, but accurate measurement of circulating TGF $\beta$ is complicated by the high TGF $\beta$ content of platelets which can release TGF $\beta$ ex vivo. We evaluated the use of citrate-theophylline-adenosinedipyridamole (CTAD) tubes to reduce preanalytical variation in TGF $\beta$ measurements caused by ex vivo platelet activation. CTAD substantially reduced ex vivo platelet activation relative to traditional plasma collections in normal donors, which correlated with a decrease in measured TGF $\beta$ levels. We show that TGF $\beta$ levels are elevated in the majority of cancer patients with skeletal metastases, and that within-patient variability of these levels is relatively low over several weeks. Patients with elevated TGF $\beta$ could be subdivided into groups with or without evidence of platelet contribution to measured TGF $\beta$ levels. The use of CTAD tubes allows a better determination of a patient's TGF $\beta$ status, and may improve classification of patients with oncologic disease.
\end{abstract}

Keywords: TGF $\beta$, cancer, biomarker, CTAD

\section{Primary objective}

Transforming growth factor $\beta$ (TGF $\beta$ ) is a dimeric $25 \mathrm{kD}$ cytokine synthesized by multiple cell types. Three isoforms exist (TGF $\beta 1$, TGF $\beta 2$, and TGF $\beta 3$ ), of which TGF $\beta 1$ is the dominant form in humans. (For the remainder of this report, TGF $\beta$ will refer to TGF $\beta 1$ unless otherwise specified). Although the majority of tissues in the human body are capable of synthesizing TGF $\beta$ under appropriate conditions, two of the tissues with the highest TGF $\beta$ content in normal donors are platelets $(20 \mathrm{mg} / \mathrm{kg})$ (Assoian and Sporn 1986) and bone (200 $\mu \mathrm{g} / \mathrm{kg}$ ) (Seyedin et al 1985). The role of TGF $\beta$ in normal biology is intricate and depends largely on the environment in which TGF $\beta$ is secreted. These actions can include suppression of cell proliferation, enhanced extracellular matrix deposition, and immunomodulatory effects (Blobe et al 2000). The role of TGF $\beta$ in cancer is even more complex, where TGF $\beta$ has been proposed to have roles as both a tumor suppressor in the premalignant phase of disease and as a pro-oncogenic factor in later stage metastatic disease (Roberts and Wakefield 2003; Siegel and Massague 2003). Because of this, TGF $\beta$ may play an important role in the pathological processes of cancer.

The use of TGF $\beta$ as a biomarker in cancer has been explored for years with varying success. Immunohistochemical analysis of biopsy material has shown increased TGF $\beta$ staining in many different tumor types (Gordinier et al 1999; Nio et al 2005), which correlated to clinical parameters such as metastasis and disease progression in some studies (Hazelbag et al 2004; Yamamoto et al 2004; Culhaci et al 2005). Because TGF $\beta$ is readily secreted by most cell types, a number of groups have evaluated the use of circulating TGF $\beta$ levels as a potential cancer biomarker. Results from such approaches have been variable, with some reports suggesting that 
circulating TGF $\beta$ levels have clinical utility in predicting disease progression (Shariat et al 2001), while others show no relationship between circulating TGF $\beta$ and outcome (Wakefield et al 1995).

One factor that complicates the use of circulating TGF $\beta$ levels as a biomarker is the potential for ex vivo release of TGF $\beta$ from platelet stores due to platelet activation. It has long been appreciated that platelets are a rich source of TGF $\beta$ (Assoian and Sporn 1986), and that as a result serum measurements of TGF $\beta$ are largely driven by platelet count and do not accurately reflect true circulating levels of TGF $\beta$ (Kropf et al 1997). As a result, plasma is the preferred matrix for TGF $\beta$ assessment. However, some level of platelet activation occurs even with routine plasma collection, and the amount of ex vivo activation can vary significantly depending upon the type of anticoagulant tube used (Golanski et al 1996; Neufeld et al 1999; Philippe et al 2004). For this reason, methods to reduce the level of platelet activation in plasma samples would be useful to help limit overestimation of in vivo TGF $\beta$ levels due to $e x$ vivo activation of platelets.

In this study, we have evaluated the use of citratetheophylline-adenosine-dipyridamole (CTAD) tubes as a means to provide more accurate TGF $\beta$ measurements in platelet-poor plasma from cancer patients. CTAD tubes contain citrate anticoagulant and a mixture of theophylline, adenosine, and dipyramidole, which serve to reduce $e x$ vivo platelet activation by increasing cAMP and inhibiting calcium-mediated platelet activation (Contant et al 1983; Macey et al 2002). We have characterized the effects of this matrix on a commonly used TGF $\beta$ ELISA, and applied this approach to the measurement of TGF $\beta$ in healthy volunteers and in oncology patients with skeletal metastases. We demonstrate that CTAD tubes are an acceptable matrix for TGF $\beta$ analysis, and that the reduction of ex vivo platelet activation using these tubes leads to a concomitant reduction in measured TGF $\beta$ levels in healthy volunteers. Using this technique we demonstrate that the majority of oncology patients with skeletal metastases have elevations in circulating TGF $\beta$ levels, and that the within-patient variability of these measurements are relatively low over a two-week period. In addition, the use of CTAD plasma coupled with measurement of platelet activation markers allows differentiation of patients with or without substantial platelet contributions to measured TGF $\beta$ levels. This approach is an extension of the previous works showing that control of platelet activation is critical to the assessment of circulating TGFb levels (Reinhold et al 1997).

\section{Methods and procedures}

\section{Patient population}

After obtaining Institution Review Board approval of the clinical protocol and informed consent from patients, samples were collected from oncology patients from three different institutions as part of an ongoing clinical trial designed to examine TGF $\beta$-related biomarkers (Baselga et al 2008). Patients were enrolled if they had previously-documented cancer involving skeletal metastases. 49 patients participated in this study, and CTAD tubes were collected 4 times over a 2-4 week period with no limitations on state at collection (diurnal, fasted.fed, etc). Patients received no oncolytic therapies during this period. Tumor types in this study included breast cancer (23 patients), prostate cancer (15) multiple myeloma (7), renal cell carcinoma (3), and lymphoma (1).

\section{Sample collection and TGF $\beta$ I analysis}

Samples were collected into either tripotassium ethylenediamine tetra-acetate ( $\mathrm{K}_{3}$ EDTA) or CTAD tubes (Becton, Dickinson and Co, Franklin Lakes, NJ). A modified protocol was used for these collections. Briefly, a tourniquet was initially used to locate the vein, then removed before collection. After removal of the tourniquet, a single discard tube was drawn to allow washout of any activation products caused by initiation of phlebotomy, followed by the CTAD tube. Tubes were then spun for $15 \mathrm{~min}$ at $2500 \mathrm{~g}$, and the central portion of the plasma layer transferred to a second tube. This tube was then spun again for $15 \mathrm{~min}$ at $2500 \mathrm{~g}$, and the supernatant transferred to a final tube for storage at $-70{ }^{\circ} \mathrm{C}$ until analysis.

TGF $\beta$ levels were analyzed using a commercially available ELISA (R\&D Systems, Minneapolis, MN, catalog \# DB100) per the manufacturer's protocol. Briefly, plasma samples underwent initial acidification/neutralization steps immediately prior to analysis to activate latent TGF $\beta$ to an immunoreactive form, under conditions which have been shown to minimize re-association with TGF $\beta$ binding proteins (Kropf et al 1997). The ELISA assay utilized soluble TGF $\beta$ type II receptor coated on the ELISA plate for antigen capture, with detection via a HRP-linked polyclonal specific for TGF $\beta 1$. Recombinant human TGF $\beta 1$ (rhTGF $\beta$ ) included in the kit was used as a standard and for the spiking experiments that follow below.

Analytical performance of the TGF $\beta 1$ assay in CTAD matrix was evaluated as follows: Assay precision was determined using 4 samples ranging from $0.05 \mathrm{ng} / \mathrm{ml}$ to $15.8 \mathrm{ng} / \mathrm{ml}$, which were run in triplicate in 5 runs over a 3 day period. Within- and between-day variance were expressed 
as the coefficient of variation (standard deviation/mean) for these samples. Matrix effects were assessed by spiking known concentrations of the rhTGF $\beta$ standard into CTAD samples, and evaluating recovery relative to buffer controls. Dilutional linearity was determined by performing a dilution series ranging from 1:2 to 1:20. Dilutions were run in triplicate, and the percent variation in observed values compared to expected results was calculated for each dilution. Freeze-thaw stability was assessed using 4 aliquots of samples stored at $-70{ }^{\circ} \mathrm{C}$ which were subjected to between 1 and 4 freeze-thaw cycles. All 4 aliquots were then assayed in triplicate in a single run.

Clinical trial samples were stored at $-70{ }^{\circ} \mathrm{C}$ until the conclusion of the study, then batch analyzed for TGF $\beta$ levels. In addition to TGF $\beta$, separate aliquots from these same CTAD plasma samples were also batch analyzed for two markers of platelet activation, platelet factor 4 (PF4) and beta-thromboglobulin (BTG) using commercial enzyme immunoassays (Asserachrom ${ }^{\circledR}$ PF4 and Asserachrom ${ }^{\circledR}$ BTG, both from Diagnostica Stago, Asnieres-Sur-Siene, France).

\section{Main outcomes and results Evaluation of CTAD matrix for TGF $\beta$ measurements}

We initially examined the analytical performance of the TGF $\beta 1$ ELISA assay in CTAD plasma to ensure that this matrix did not adversely affect assay performance. As shown in Table 1, analytical parameters for the TGF $\beta 1$ assay in CTAD plasma were comparable to that reported for EDTA plasma by the manufacturer (Table 1). Withinand between-day coefficient of variation (CV) ranged from $5.9 \%-13.4 \%$ and $9.1 \%-29 \%$, respectively, with the highest variability seen at $0.05 \mathrm{ng} / \mathrm{ml}$. The $0.05 \mathrm{ng} / \mathrm{ml}$ concentration was below manufacturer's recommendations, but was initially evaluated in an attempt to extend the reportable range of the assay in the expectation that TGF $\beta$ levels in CTAD plasma would be lower than reported for EDTA plasma due to decreased platelet activation. No evidence of a matrix effect was seen in either the recovery or dilutional linearity experiments, with less than $10 \%$ variation from expected results at most concentrations. Freeze-thaw stability of TGF $\beta$ was observed for two freeze-thaw cycles in CTAD with $<10 \%$ loss of signal, but further freeze-thaw cycles led to a progressive loss of signal $(>15 \%$ change from original values).

\section{Impact of CTAD tubes on ex vivo platelet activation and TGF $\beta$ measurement}

Normal donor samples were next used to evaluate the differences between EDTA and CTAD tubes on ex vivo TGF $\beta$ release, and to generate an estimate of the reference range for TGF $\beta$ in the CTAD matrix. Paired EDTA and CTAD tubes were drawn from 10 normal donors and evaluated for TGF $\beta$ as well as two markers of platelet activation, platelet factor 4 and beta-thromboglobulin. PF4 and BTG are contained in platelet alpha granules in approximately equimolar amounts (Kaplan and Owen 1981), and are released following platelet activation or platelet lysis. In our study, BTG and PF4 levels were both elevated in EDTA tubes relative to CTAD for all donors, confirming that CTAD confers an advantage in limiting ex vivo platelet activation relative to EDTA (Figure 1A). A concurrent increase in TGF $\beta$ levels was also seen in the EDTA tubes from all donors, resulting in a $67 \%-430 \%$ bias in TGF $\beta$ levels in the EDTA plasma relative to the CTAD plasma (Figure 1B). An additional 15 CTAD samples were then drawn from normal donors

Table I Analytical performance of TGF $\beta$ ELISA in CTAD matrix

\begin{tabular}{|c|c|c|c|c|}
\hline & & & CTAD & EDTA \\
\hline \multirow[t]{4}{*}{ Precision } & Within-day & Concentrations tested $(\mathrm{ng} / \mathrm{ml})$ & $0.05,0.2,8.0,15.8$ & $0.88,5.14, \mathrm{I} I .13$ \\
\hline & & $\mathrm{CV}$ & |3.4\%, 8.3\%, 5.8\%, 5.9\% & $7.3 \%, 4.9 \%, 3.7 \%$ \\
\hline & Between-day & Concentrations tested $(\mathrm{ng} / \mathrm{ml})$ & $0.05,0.2,8.0,15.8$ & $\mathrm{I} .18,4.23,7.21$ \\
\hline & & $\mathrm{CV}$ & $29 \%, 10.8 \%, 11.5 \%, 9.1 \%$ & $12.7 \%, 10.3 \%, 9.8 \%$ \\
\hline Reportable range & & & $0.75-240 \mathrm{ng} / \mathrm{ml}$ & $0.36-240 \mathrm{ng} / \mathrm{ml}$ \\
\hline \multirow[t]{2}{*}{ Dilutional linearity } & & Dilutions tested & $\mathrm{I}: 2, \mathrm{I}: 5, \mathrm{I}: \mathrm{I} 0, \mathrm{I}: 20$ & $\mathrm{I}: 2, \mathrm{I}: 4$ \\
\hline & & $\%$ of expected value & $100 \%, 98 \%, 97 \%, 120 \%$ & $103 \%, 103 \%$ \\
\hline Freeze-thaw stablility & & & Stable to 2 freeze/thaw cycles & NR \\
\hline
\end{tabular}

Notes: Experiments were performed as described in methods. CVi and \% values are listed in order corresponding to tested concentrations for all experiments. Performance parameters in EDTA were taken from the manufacturer's package insert for the TGF $\beta$ ELISA. The analytical performance of the assay in CTAD plasma was comparable with that reported for EDTA plasma.

Abbreviations: CTAD, citrate-theophylline-adenosine-dipyridamole; CVi, within-patient variability; EDTA, ethylenediamine tetra-acetate; NR, not reported;TGF $\beta$, transforming growth factor beta. 
A

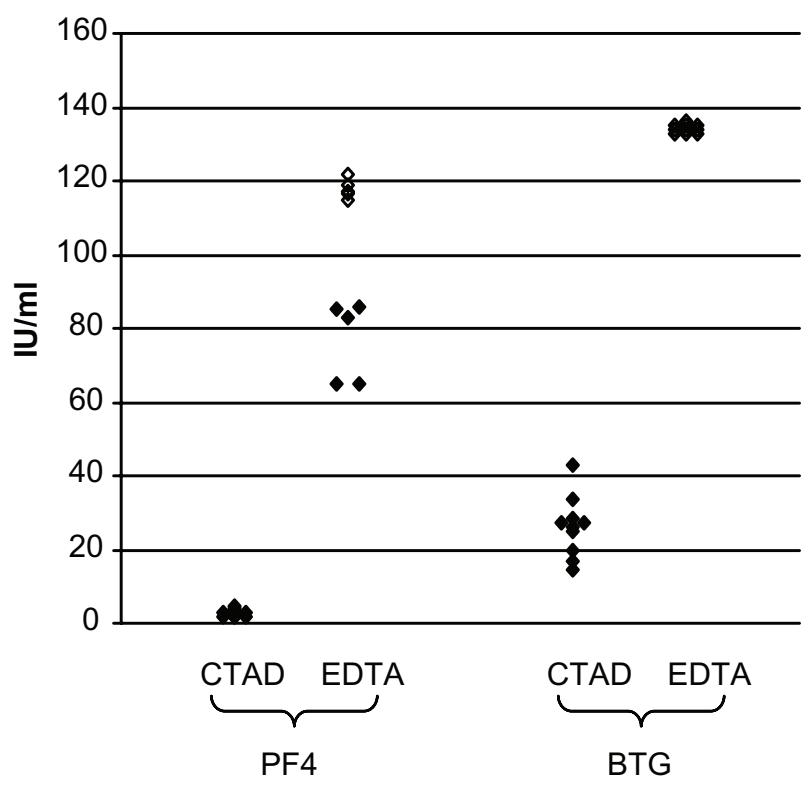

B

TGFb levels

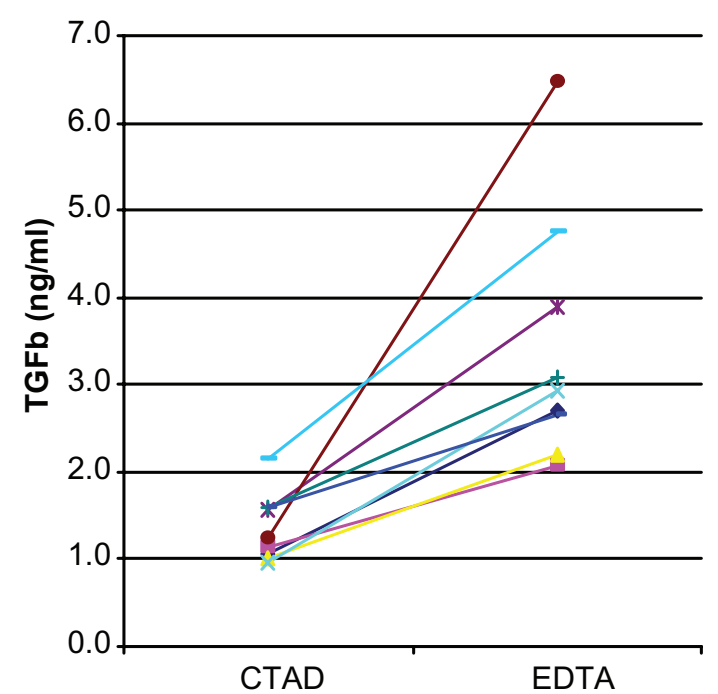

Figure I Effect of CTAD matrix on platelet activation and TGF 3 measurements. A) Markers of platelet activation. Both PF4 and BTG were substantially elevated in samples collected in EDTA tubes from normal donors, confirming that some level of ex vivo platelet activation occurs in EDTA plasma draws. (Open symbols represent values outside the range of the assay). B) Within-donor TGF $\beta$ levels in CTAD and EDTA tubes. EDTA TGF $\beta$ levels were higher than CTAD tube levels for all donors, ranging from I.7- to 5.3-fold higher.

Abbreviations: BTG, beta-thromboglobulin; CTAD, citrate-theophylline-adenosine-dipyridamole; EDTA, ethylenediamine tetra-acetate; PF4, platelet factor 4;TGF $\beta$, transforming growth factor beta.

to estimate the reference range for TGF $\beta$ in this matrix. The median TGF $\beta$ level from all 25 samples was $1.3 \mathrm{ng} / \mathrm{ml}$ (range $0.8-2.2 \mathrm{ng} / \mathrm{ml}$ ), with a 95th percentile of $1.7 \mathrm{ng} / \mathrm{ml}$, which was established as the upper limit of normal for the following oncology study.

\section{Levels and variability of TGF $\beta$ measurements in oncology patients with advanced disease}

A total of 188 samples were collected in CTAD tubes from 49 oncology patients, and TGF $\beta$, PF4 and BTG levels were measured as described. A surprising observation in this study was that 118 samples (63\%) showed evidence of platelet activation, despite the use of CTAD tubes. There was good correlation between the two platelet activation markers in individual samples, with the majority of samples showing agreement between PF4 and BTG (Figure 2A). TGF $\beta$ levels were significantly increased in the samples from oncology patients relative to normal donors ( $\mathrm{p}<0.05$, t-test), with $77 \%$ of the samples collected falling above the upper limit of normal established in the healthy donor population. As one would expect, samples with evidence of platelet activation showed the highest levels of TGF $\beta$ (Figure 2B). However, TGF $\beta$ levels were also elevated in approximately half of the samples with normal PF4 and BTG levels, suggesting that in those patients TGF $\beta$ elevation may be driven by a mechanism independent of platelet release (such as elevated in vivo production of TGF $\beta$ from another source).

We next considered the within-patient variability (CVi) of TGF $\beta$ levels in the 47 patients who had at least 3 samples collected. The median CVi was relatively low for these patients $(22.7 \%)$, with the majority of patients showing $<40 \%$ variation in TGF $\beta$ levels over the two week period tested (Figure 3A). In addition to this consistency in TGF $\beta$ levels, an interesting finding was that many patients also showed consistent patterns of platelet activation markers over repeated visits. The variability of TGF $\beta$ measurements was lowest in the patients who had consistently normal PF4 and BTG levels, while high CVi values were more common in the patients showing evidence of platelet activation. This increased variability in the platelet-activated subset was not simply attributable to an increase in measured TGF $\beta$ levels, as many patients with high TGF $\beta$ levels showed relatively low CVi (Figure 3B).

\section{Conclusions}

In this study we have shown that CTAD tubes are a suitable anticoagulant for TGF $\beta$ analysis, and that a commercially available TGF $\beta 1$ ELISA assay shows acceptable 
A

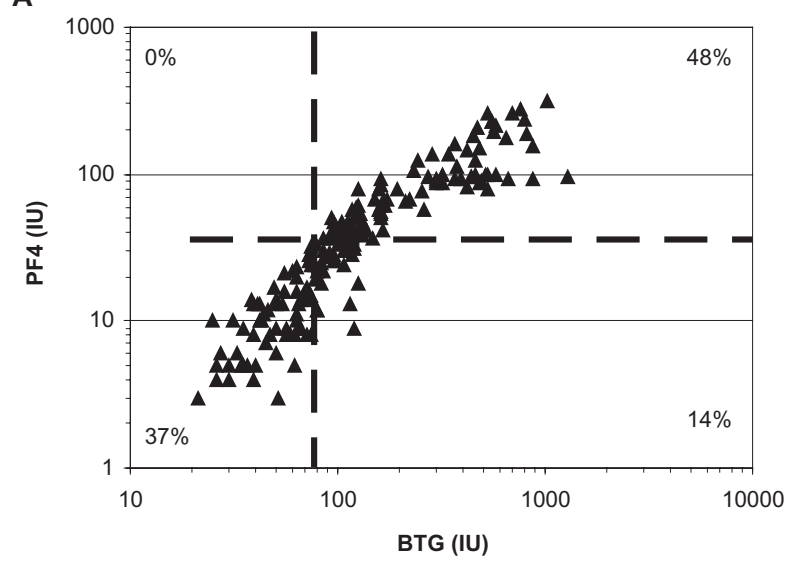

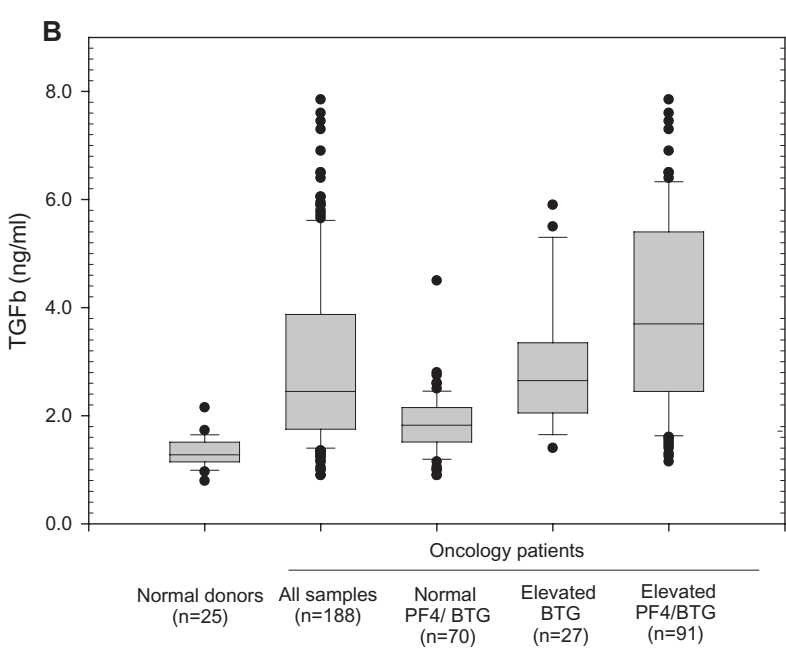

Figure 2 CTAD plasma TGF $\beta$ levels in normal donors and oncology patients. A) Relationship of platelet-activation markers in clinical samples. Dashed lines represent the upper limit of normal for both markers, \%'s indicated fraction of all samples falling in each quadrant. The overall correlation between platelet activation markers was good across all samples ( $r^{2}=0.7$ I, Pearson correlation coefficient), with agreement between PF4 and BTG in $85 \%$ of the samples. B) TGF $\beta$ levels in oncology patients related to platelet activation. Boxes represent the median and central 50th percentile of observations, whiskers represent the 10th and 90th percentiles with individual outliers noted, dashed line represents upper limit established in normal donors ( $1.7 \mathrm{ng} / \mathrm{ml}$ ).A single sample with an extremely elevated TGF $\beta$ level (I3.2 pg/ml, BTG/PF4 +ve) is not shown to improve scaling. Samples collected from oncology patients had significantly higher circulating TGF $\beta$ levels than those from normal donors ( $p<0.05$ students t-test for all groups when compared to normal donors). This was true regardless of platelet activation status, although TGF $\beta$ levels were higher in samples with evidence of platelet activation.

Abbreviations: BTG, beta-thromboglobulin; CTAD, citrate-theophylline-adenosine-dipyridamole; PF4, platelet factor 4;TGF $\beta$, transforming growth factor beta.

performance characteristics in this matrix. As predicted, the use of CTAD tubes led to a decreased level of ex vivo platelet activation when compared to traditional plasma collections in $\mathrm{K}_{3} \mathrm{EDTA}$, which corresponded to a decrease in measured TGF $\beta$ levels in normal donors. The contribution of ex vivo platelet activation on TGF $\beta$ levels in normal donors was substantial in EDTA, resulting in as much as a 5-fold increase in measured TGF $\beta$ in these samples. We have seen similar results with VEGF analysis in CTAD vs EDTA tubes (data not shown), and a recent publication has shown similar results for a number of platelet-derived growth factors including TGF $\beta$ (Zimmermann et al 2005), suggesting that this approach may be relevant to the measurement of many circulating cytokines.

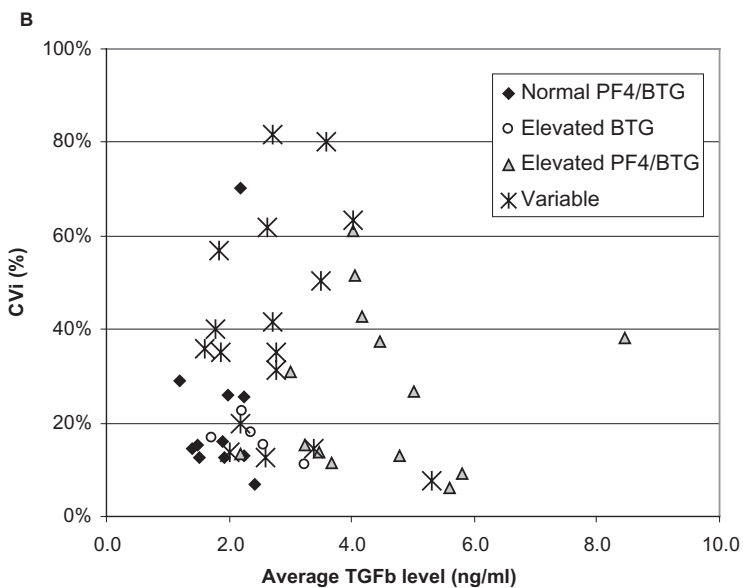

Figure 3 Within-patient variability (CVi) of TGF $\beta$ measurements in oncology patients. Boxes represent the median and central 50 th percentile of observations, whiskers represent the 10th and 90th percentiles with individual outliers noted. A) CVi of repeated measurement calculated for all patients with at least 3 visits. Subgroups are defined as patients who had the specified pattern in $\geq 3$ of 4 visits. Variability was lowest in the patients with consistent patterns of platelet activation markers. B) Relationship between within-patient variability and TGF $\beta$ levels. $X$ axis represents average TGF $\beta$ level across all visits for each patient. There was no correlation between CVi and TGF $\beta$ levels for any of the groups.

Abbreviations: BTG, beta-thromboglobulin; CTAD, citrate-theophylline-adenosine-dipyridamole; EDTA, ethylenediamine tetra-acetate; PF4, platelet factor 4;TGF $\beta$, transforming growth factor beta. 
TGF $\beta$ levels were elevated in the majority of the oncology patients enrolled in this trial. This was not entirely unexpected, as enrollment was limited to patients with skeletal metastases which is a population where TGF $\beta$ has been shown to play a significant role (Kang et al 2005). There were substantial differences in TGF $\beta$ levels between patients, with average levels over the 4 visits ranging from 1.2-8.5 $\mathrm{ng} / \mathrm{ml}$ across the groups of patients enrolled. However, even with this wide range of TGF $\beta$ values across the population, within-patient variability was relatively low in most patients. This is encouraging for potential use of TGF $\beta$ as a biomarker, because it implies that limited sampling would be required to accurately assess a patient's TGF $\beta$ status. We believe that the use of CTAD tubes contributed to this consistency, by reducing the variability that would be introduced by sporadic ex vivo platelet activation during the collection process. This is supported by the observation that the subset of patients where PF4 and BTG were sporadically elevated showed the highest levels of within-patient variability.

A surprising observation in this study was that a large number of samples showed elevated levels of BTG and PF4 despite the use of CTAD tubes to limit ex vivo platelet activation. The cause of this is not clear. One possibility is that this represents ex vivo platelet activation, either due to poor sample processing or an inherent increase in platelet reactivity in some oncology patients. Alternatively, the elevations in PF4 and BTG may indicate an increased in vivo platelet activation in a subset of these oncology patients. This would explain the within-patient consistency of activation marker patterns across multiple visits, and could reflect the hypercoagulable state commonly found in patients with advanced cancer (Caine et al 2002). It is interesting to note that patients with elevated TGF $\beta$ levels in this study can be largely divided into two groups using these platelet markers. Patients with repeated evidence of platelet activation showed the highest levels of TGF $\beta$, consistent with the observation that platelets contain large amounts of TGF $\beta$. However, an additional group of patients had elevations of TGF $\beta$ in the absence of any evidence of platelet activation. It is possible that these patients represent a subset where TGF $\beta$ secretion from the tumor milieu is the primary driver of elevated circulating levels. Further work will be required to define if there is any biological relevance to differentiating between these subgroups in terms of predicting either the disease prognosis or response to TGF $\beta$-targeted therapies.

In summary, we believe that the use of CTAD tubes coupled with PF4 and BTG measurements will improve the ability to accurately assess a patient's true TGF $\beta$ status by reducing the background noise introduced by $e x$ vivo platelet activation. Removing a potential source of preanalytical error should reduce the inherent variability of TGF $\beta$ measurements in patients, and allow for a better evaluation of the true utility of circulating TGF $\beta$ levels as a cancer biomarker. Further work applying this technique to well-annotated clinical specimens will be required to determine the value of circulating TGF $\beta$ levels as a potential prognostic tool.

\section{Disclosure}

The authors report no conflicts of interest in this work.

\section{References}

Assoian RK, Sporn MB. 1986. Type beta transforming growth factor in human platelets: release during platelet degranulation and action on vascular smooth muscle cells. J Cell Biol, 102:1217-23.

Baselga J, Rothenberg ML, Tabernero J, et al. TGF- $\beta$ signaling related markers in cancer patients with bone metastasis. Biomarkers, 13:217-36.

Blobe GC, Schiemann WP, Lodish HF. 2000. Role of transforming growth factor beta in human disease. N Engl J Med, 342:1350-8.

Caine GJ, Stonelake PS, Lip GY, et al. 2002. The hypercoagulable state of malignancy: pathogenesis and current debate. Neoplasia (New York), 4:465-73.

Contant G, Gouault-Heilmann M, Martinoli JL. 1983. Heparin inactivation during blood storage: Its prevention by blood collection in citric acid, theophylline, adenosine, dipyridamole-C.T.A.D. mixture. Thromb Res, 31:365-74.

Culhaci N, Sagol O, Karademir S, et al. 2005. Expression of transforming growth factor-beta-1 and $\mathrm{p} 27 \mathrm{Kip} 1$ in pancreatic adenocarcinomas: relation with cell-cycle-associated proteins and clinicopathologic characteristics. BMC Cancer, 5:98.

Golanski J, Pietrucha T, Baj Z, et al. 1996. Molecular insights into the anticoagulant-induced spontaneous activation of platelets in whole blood - various anticoagulants are not equal. Thromb Res, 83:199-216.

Gordinier ME, Zhang HZ, Patenia R, et al. 1999. Quantitative analysis of transforming growth factor beta 1 and 2 in ovarian carcinoma. Clin Cancer Res, 5:2498-505.

Hazelbag S, Kenter GG, Gorter A, et al. 2004. Prognostic relevance of TGFbeta1 and PAI-1 in cervical cancer. Int J Cancer, 112:1020-8.

Kang Y, He W, Tulley S, et al. 2005. Breast cancer bone metastasis mediated by the Smad tumor suppressor pathway. Proc Natl Acad Sci USA, 102:13909-14.

Kaplan KL, Owen J. 1981. Plasma levels of beta-thromboglobulin and platelet factor 4 as indices of platelet activation in vivo. Blood, 57:199-202.

Kropf J, Schurek JO, Wollner A, et al. 1997. Immunological measurement of transforming growth factor-beta 1 (TGF- $\beta 1$ ) in blood; assay development and comparison. Clin Chem, 43:1965-74.

Macey M, Azam U, McCarthy D, et al. 2002. Evaluation of the anticoagulants EDTA and citrate, theophylline, adenosine, and dipyridamole (CTAD) for assessing platelet activation on the ADVIA 120 hematology system. Clin Chem, 48:891-9.

Neufeld M, Nowak-Gottl U, Junker R. 1999. Citrate-theophylline-adeninedipyridamol buffer is preferable to citrate buffer as an anticoagulant for flow cytometric measurement of platelet activation. Clin Chem, 45:2030-3.

Nio Y, Omori H, Hashimoto K, et al. 2005. Immunohistochemical expression of receptor-tyrosine kinase c-kit protein and TGF-beta1 in invasive ductal carcinoma of the pancreas. Anticancer Res, 25:3523-9.

Philippe J, De Logi E, Baele G. 2004. Comparison of five different citrated tubes and their in vitro effects on platelet activation. Clin Chem, 50:656-8. 
Reinhold D, Bank U, Buhling F, et al. 1997. A detailed protocol for the measurement of TGFb1 in human blood samples. J Immun Methods, 209:203-6.

Roberts AB, Wakefield LM. 2003. The two faces of transforming growth factor beta in carcinogenesis [comment]. Proc Natl Acad Sci USA, 100:8621-3.

Seyedin SM, Thomas TC, Thompson AY, et al. 1985. Purification and characterization of two cartilage-inducing factors from bovine demineralized bone. Proc Natl Acad Sci USA, 82:2267-71.

Shariat SF, Shalev M, Menesses-Diaz A, et al. 2001. Preoperative plasma levels of transforming growth factor beta(1) (TGF-beta(1)) strongly predict progression in patients undergoing radical prostatectomy. J Clin Oncol, 19:2856-64.
Siegel PM, Massague J. 2003. Cytostatic and apoptotic actions of TGF-beta in homeostasis and cancer. Nature Rev Cancer, 3:807-21.

Wakefield LM, Letterio JJ, Chen T, et al. 1995. Transforming growth factorbeta 1 circulates in normal human plasma and is unchanged in advanced metastatic breast cancer. Clin Cancer Res, 1:129-36.

Yamamoto T, Akisue T, Marui T, et al. 2004. Expression of transforming growth factor beta isoforms and their receptors in malignant fibrous histiocytoma of soft tissues. Clin Cancer Res, 10:5804-7.

Zimmermann R, Koenig J, Zingsem J, et al. 2005. Effect of specimen anticoagulation on the measurement of circulating platelet-derived growth factors. Clin Chem, 51:2365-8. 
